\title{
Muscle Activation Patterns in Wheelchair Basketball Athletes with and without Physical Disability
}

\section{Matthew Hanks and Gretchen Oliver*}

School of Kinesiology, Auburn University, USA

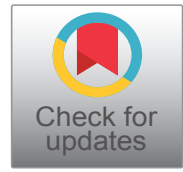

*Corresponding author: Gretchen Oliver, PhD, FACSM, ATC, CES, School of Kinesiology, Auburn University, 301 Wire Rd, Auburn AL, 36849, USA, Tel: +859-200-4035, E-mail: goliver@auburn.edu

\begin{abstract}
Wheelchair basketball is a sport designed for individuals with permanent lower body disabilities. While the sport was developed for those with physical disabilities, leagues allow individuals without a disability to play the sport. The purpose of this study was to identify muscle activations of commonly used lumbopelvic-hip complex (erector spinae, rectus abdominis, and latissimus dorsi), lower extremity (gastrocnemius and rectus femoris), and upper extremity (anterior deltoid, triceps brachii, and flexor carpi radialis) musculature in wheelchair basketball free throw shooting to better understand energy transfer in wheelchair basketball players with disabilities compared to basketball players without disabilities shooting from a wheelchair. We hypothesized that muscle activations would be different between participants with disabilities and those without disabilities, and that participants with disabilities would have greater trunk and upper extremity activations due to altered mechanics. Results of this study showed greater muscle activation (\% Static Posture Assessment, \%SPA) of the rectus abdominis (608.0 vs. $195.0 \%$ SPA, $p=0.025$ ), latissimus dorsi (446.0 vs. 224.3 $\%$ SPA, $p=0.022$ ), anterior deltoid (300.3 vs. $173.7 \%$ SPA, $p=0.003$ ) and triceps brachii (1537.7 vs. $832.7 \%$ SPA, $p$ $=0.031$ ), with participants with disabilities yielding greater muscle activation in the four aforementioned muscles compared to the participants without disabilities. Results of this research identified greater activation in muscles commonly used in wheelchair basketball shooting in athletes with disabilities. These data could provide strategies for coaches and strength and conditioning staff members to implement strength and conditioning programs to further increase activation and efficiency in these muscles to increase performance outcomes, such as free throw shooting percentage.
\end{abstract}

\section{Keywords}

Adaptive sports, Electromyography, Free throw

\section{Introduction}

Wheelchair basketball is a highly competitive sport that uses primarily upper extremity and lumbo-pelvic musculature to accomplish all tasks of the sport (i.e., wheelchair propulsion, dribbling, passing, shooting, and rebounding) [1]. Free throw execution is one of the basic skills first introduced when learning the sport of wheelchair basketball, and the mechanics utilized during a free throw attempt are similar to overall shooting mechanics. Free throw performance plays a pivotal role in many games because it allows an athlete an attempt to score uncontested points, free from defenders and other performance-altering situations [2]. According to Ingram and Snowden [3], the free throw is a true representation of preferred shooting mechanics making this task reliable for examining overall shooting mechanics in wheelchair basketball athletes.

While the majority of wheelchair basketball athletes are individuals with permanent lower body disabilities that prevent them from playing standing basketball, there are many standing basketball players without disabilities who play wheelchair basketball for recreation or out of support for loved ones [4]. Because both individuals with and without disabilities play this sport, it is important to understand how muscles in the human body, with and without disabilities, activate to perform shooting tasks within the sport, such as shooting a free throw.

Decreased free throw shooting percentages are common in wheelchair basketball compared to standing basketball. Malone, et al. [2] reported free throw percentages in professional standing basketball players at the 1994 Men's World Championship were 59\% to 83\%, with an average free throw shooting percentage of $71 \%$;

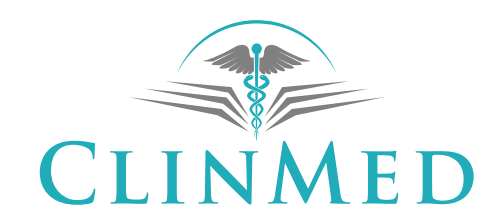

INTERNATIONAL LIBRARY
Citation: Hanks M, Oliver G (2018) Muscle Activation Patterns in Wheelchair Basketball Athletes with and without Physical Disability. Int J Physiatry 4:013. doi.org/10.23937/2572-4215.1510013 Received: September 11, 2017: Accepted: January 10, 2018: Published: January 12, 2018 Copyright: (c) 2018 Hanks M, et al. This is an open-access article distributed under the terms of the Creative Commons Attribution License, which permits unrestricted use, distribution, and reproduction in any medium, provided the original author and source are credited. 
whereas free throw percentages at the 1992 Paralympic Games in male wheelchair basketball players were 35\% to $54 \%$, with an average free throw shooting percentage of $41 \%$ [5]. More recently, the National Basketball Association (NBA) reported average free throw percentages of $70.6 \%$ to $81.5 \%$ for all teams in the $2016-2017$ regular season; [6] whereas the International Wheelchair Basketball Federation (IWBF) reported average free throw percentages of $24.6 \%$ to $100.0 \%$ for the 2017 Men's Americas Cup gold, silver, and bronze medal teams (USA, Canada, Brazil), with an average free throw percentage of $53.6 \%$ [7].

Researchers attribute these drastic differences in free throw performance to trajectory and kinematic differences between high and low functioning wheelchair basketball players $[2,5,8]$. Typically, these researchers explored shooting mechanics based on a player's IWBF classification [9], to which they are assigned due to the level of anatomical function they possess as a result of their physical disability $[2,5,8]$. Malone, et al. [2] found that players of lower functioning classifications (Class 1 and 2) adopted a strategy of shooting the ball with a steeper ball trajectory which was accomplished by using greater shoulder and elbow angular velocities to generate a more forceful shot compared to the higher functioning classifications (Class 3 and 4) who shot the ball with a more shallow approach from a higher release point [2].

Overhead shooting activities require the body to act as a kinetic chain to summate energy from the lower extremity to the upper extremity for coordinated movement, and allow for less stress and need for energy generation at the more distal segments [10-13]. However, to the authors knowledge, there has yet to be data available examining the function of the kinetic chain in wheelchair basketball players performing an overhead shooting task, therefore it is important to analyze muscle activations of the lower and upper extremities, as well as the trunk in wheelchair basketball shooting, despite the notion that physical disabilities in the lower extremities may result in diminished muscle activity. In athletes with physical disabilities, it is possible that they maintain movement in a proximal to distal sequencing fashion, despite a possible decrease in function of the lower extremity musculature. That is, the shooting movement originates in the lumbopelvic-hip complex, and distal segments (i.e., the shoulder, upper arm, forearm, and wrist) move with increasing velocity relative to the adjacent proximal segment, thereby increasing distal segment velocity and force production [12,13]. If this is true, it seems likely that the lumbopelvic-hip complex serves as the origin for kinetic chain principles in disabled athletes, and muscle activations will be different for trunk and upper extremity musculature between athletes with disabilities and athletes without disabilities.
Understanding neuromuscular activation patterns utilized in the kinetic chain during free throw performance in wheelchair basketball players can be researched using electromyography. Electromyography (EMG) provides insight into muscle activity during functional movements and can be easily translated into clinical and rehabilitative settings. The use of electromyography in examining muscle activity in standing basketball athletes is not novel; however, research using surface electromyography (sEMG) in wheelchair basketball athletes is sparse. Of studies performed using sEMG in wheelchair users, most examine muscle activation during wheelchair propulsion, focusing solely on the upper extremities [14-16]; however, there are no studies using sEMG to investigate full-body muscle activity in wheelchair basketball athletes.

Mechanics across all classifications and levels of function could be altered by trunk and upper extremity strength and conditioning protocols that would allow the player to shoot a ball with greater force and ease due to acquired strength gains; however, this was outside the scope of previous research $[2,5,8]$. It may be beneficial to use sEMG to assess commonly used musculature in wheelchair basketball players with physical disabilities compared to standing basketball players shooting a free throw from a wheelchair. Differences in muscle recruitment patterns may indicate specific needs for muscular strength and development to perform successful free throw shooting in individuals with a disability compared to individuals without a disability.

The purpose of this study was to identify muscle activations of commonly used lumbopelvic-hip complex (erector spinae, rectus abdominis, and latissimus dorsi), lower extremity (gastrocnemius and rectus femoris), and upper extremity (anterior deltoid, triceps brachii, and flexor carpi radialis) musculature in wheelchair basketball free throw shooting to better understand energy transfer in wheelchair basketball players with disabilities compared to basketball players without disabilities shooting from a wheelchair. Due to proximal-to-distal sequencing patterns found in other overhead sports $[10,12,13,17]$, we hypothesized that activations of trunk and upper extremity musculature would be greater in participants with physical disabilities and activations of lower extremity musculature would be greater in participants without a physical disability.

\section{Methods}

\section{Participants}

Forty experienced basketball athletes participated and were divided into two groups. Wheelchair basketball athletes with permanent disabilities affecting lumbopelvic-hip complex and lower extremity function were placed into one group ( $n=21 ; 24.3 \pm 4.4$ years, $8.9 \pm 4.9$ years of experience) based on the International Wheelchair Basketball Federation classification [9] and 
Table 1: Participant IWBF classification and medical diagnoses.

IWBF (International Wheelchair Basketball Federation) is the governing entity responsible for classifying wheelchair basketball athletes globally to ensure consistency and fairness during competition. Players are placed in groups based on their level of function in the vertical, forward, and sideways planes: Class 1- Little or no trunk movement in any plane, Class 2- Upper trunk rotation in the vertical plane, partial trunk movement in the forward plane, and little or no trunk movement in the sideways plane, Class 3- Complete trunk movement in the vertical and forward planes and little or no movement in the sideways plane, Class 4- Complete trunk movement in the vertical and forward planes, with complete movement to one side of the sideways plane, Class $4.5 /$ Able-bodied - Complete trunk movement in all planes [9].

\begin{tabular}{|l|l|l|}
\hline IWBF Classification & $\begin{array}{l}\text { Number of } \\
\text { participants }(\mathbf{n})\end{array}$ & Medical diagnoses \\
\hline 1 & 1 & T9 SCl-C \\
\hline 1.5 & 1 & Cerebral Palsy \\
\hline 2 & 2 & T8 SCI-C \\
\hline 2.5 & 5 & T9 SCI-I, Single BKA, Spina Bifida, Double BKA \\
\hline 3 & 1 & Spina Bifida \\
\hline 3.5 & 6 & $\begin{array}{l}\text { Double BKA, T12 SCl-I, Spina Bifida, Neurocardiogenic Syncope Dysautonomia } \\
\text { w/ small fiber neuropathy }\end{array}$ \\
\hline 4 & 3 & Single AKA, Fibula Limb Deficiency, Single FA, Unilateral lower leg dystrophy \\
\hline 4.5 & 2 & Complex Regional Pain Syndrome, Hip fusion \\
\hline AB & 19 & Normal function/no disability \\
\hline
\end{tabular}

$\mathrm{AB}=$ Able-Bodied; $\mathrm{T}$ = Thoracic Vertebra; SCI-C = Spinal Cord Injury, Complete; SCI-I = Spinal Cord Injury, Incomplete; $\mathrm{BKA}=$ Below Knee Amputation; AKA = Above Knee Amputation; FA = Foot Amputation.

basketball athletes without physical disabilities were placed into another group $(n=19 ; 26.1 \pm 6.2$ years, 14.9 \pm 6.9 years of experience) (Table 1 ). The Institutional Review Board of the university approved all testing protocols. Before data were collected, all testing procedures and expectations were explained to each participant and informed consent was obtained.

To participate in the study, participants must have met the criteria for one of the following groups: (1) Wheelchair basketball athlete with a physical disability playing in the Intercollegiate Division in the United States, or (2) Experienced basketball athlete without a physical disability and with $\geq 4$ years playing experience in high school/college. Other inclusion criteria were that the participant was between the ages of 19-45 years old, had no known upper or lower extremity injury within the past six months, and did not have an allergy to athletic tape.

\section{Procedures}

Surface Electromyography data were collected via a Noraxon Myopac 1400 L eight-Channel Amplifier (Noraxon USA, INC, Scottsdale, AZ). Adhesive BIO Protech bipolar, $\mathrm{Ag} / \mathrm{AgCl}$ 4-cm diameter disk-shaped surface electrodes (BIO Protech Inc., Tustin, CA) were used. The same investigator performed electrode placement, as well as data collection and processing. Raw sEMG signal was full wave rectified and smoothed based on the smoothing algorithms of root mean square at $100 \mathrm{~ms}$. Data were sampled at a rate of $1,500 \mathrm{~Hz}$ and recorded in Microvolts $(\mu \mathrm{V})$. Data from each muscle were normalized and expressed as a relative percent contribution of a static posture assessment (\%SPA) [18]. Non-shooting/ contralateral medial gastrocnemius and rectus femoris, and shooting/ipsilateral rectus abdominis, inferior erector spinae, latissimus dorsi, anterior deltoid, medial head of the triceps brachii, and flexor carpi radialis muscles were identified through palpation of the muscle belly, shaved, abraded, and cleaned using standard medical alcohol swabs for electrode placement. Following dermal preparation, surface electrodes were attached over the muscle bellies and positioned parallel to muscle fibers using previously published standardized methods $[17,19]$. The selected interelectrode distance was $25 \mathrm{~mm}$ due to slight overlap of the electrodes to account for the small muscle belly of the investigated musculature. Following electrode placement, participants were given a self-determined amount of time, not to exceed 10 minutes, to warm-up and acclimate themselves to the wheelchair (maneuvering, dribbling, and shooting). All participants without physical disabilities used the same, standardized wheelchair, whereas participants with physical disabilities were permitted to use their personal basketball wheelchair for safety and familiarity. Depending on the gender of the athlete, males used a Mikasa BD2000 men's official 29.5" ball (650 g), and females used a Mikasa BD2000 women's compact 28.5" ball (550 g) (Mikasa USA, Brea, CA). Once participants deemed themselves ready, they were instructed to position him or herself in the wheelchair at the free throw line of a standard, NCAA-regulation sized basketball court (15 ft from the hoop). Each participant was instructed to maintain a static posture of desired shot mechanics at the free throw line for 5 seconds. This position was to be similar to the instance just before ball release during a shot, to which all surface electromyography (sEMG) test trials were normalized within the participant [18]. This method was utilized in place of Maximal Volitional Isometric Contractions (MVIC) since participants with physical disabilities are unable to perform maximal isometric contractions due to neuromuscular dysfunction (i.e., paralysis, neurodegenerative diseases, and amputations). Halaki and Ginn have suggested this method to normalize the EMG signals to the mean 
activation levels obtained over the course of the task under investigation [18]. Upon completion of the static posture recording, participants began test trial attempts at a self-selected pace.

Each test trial started with a verbal cue from the researcher prompting the participant to perform the task. The participant was instructed to shoot the basketball with the goal of obtaining a 'swish', that is, the ball went cleanly through the hoop with no contact on the rim. The use of a 'swish' is validated by previous research identifying it as the most common execution of the free throw using the most accurate mechanics [2]. Participants were instructed to make a total of three 'swish' shots from the free throw line. They were allowed as many attempts necessary to successfully make three 'swish' shots. Missed shots were rebounded for them, so they could maintain in position at the free throw line, as well as limit the onset of fatigue due to wheelchair propulsion. For the EMG analysis, the shot was defined as one phase (shooting phase) between two events. The shooting phase was defined as the first instance of upward movement of the shooting arm (event one) until the cessation of their follow-through (first instance of downward movement of the shooting arm; event two). One researcher analyzed all data and gave instructions for all data collections to remain consistent for the duration of the study.

\section{Statistical analysis}

Data were analyzed using Statistical Package for Social Science (SPSS) software (version 23; SPSS Inc., Chicago, IL, USA). Normalized EMG data from eight muscles: Contralateral gastrocnemius and rectus femoris, and ipsilateral inferior erector spinae, rectus abdominis, latissimus dorsi, anterior deltoid, triceps brachii, and flexor carpi radialis were analyzed. Due to a non-normal distribution of the raw data (Figure 1a), natural log transformations were made to fit a normal distribution (Figure 1c). Data presented are the raw data (Figure 1b) and the natural log of the raw data (Figure $1 \mathrm{~d}$ ), that is, a percentage of the SPA, originally measured in $\mu \mathrm{V}$. This process normalized EMG values and modified outliers to fit within a normal distribution. Shapiro-Wilk's Test for Normality revealed normality of log-transformed data. Mauchly's Test revealed a violation of the assumption of sphericity, thus within-subjects effects were observed using a Greenhouse-Geisser correction. Levene's Test for the Homogeneity of Variances was tested and found to uphold the assumption of equal variances in the log-transformed data. An independent samples t-test was used to compare mean differences in muscle activation between groups (Disability vs. No Disability) in the following muscles: Gastrocnemius, rectus femoris, erector spinae, rectus abdominis, latissimus dorsi, anterior deltoid, triceps brachii, and flexor carpi radialis. Significant results obtained from the log-transformed independent samples t-test agreed with a Mann-Whitney $U$ test for the non-normally distributed raw data further validating these findings. The alpha level was set a priori at $\mathrm{p}=0.05$ using Bonferroni corrections for all analyses.

\section{Results}

Mann-Whitney $U$ test using non-parametric data found significant median differences in muscle activation between participants with and without disabilities

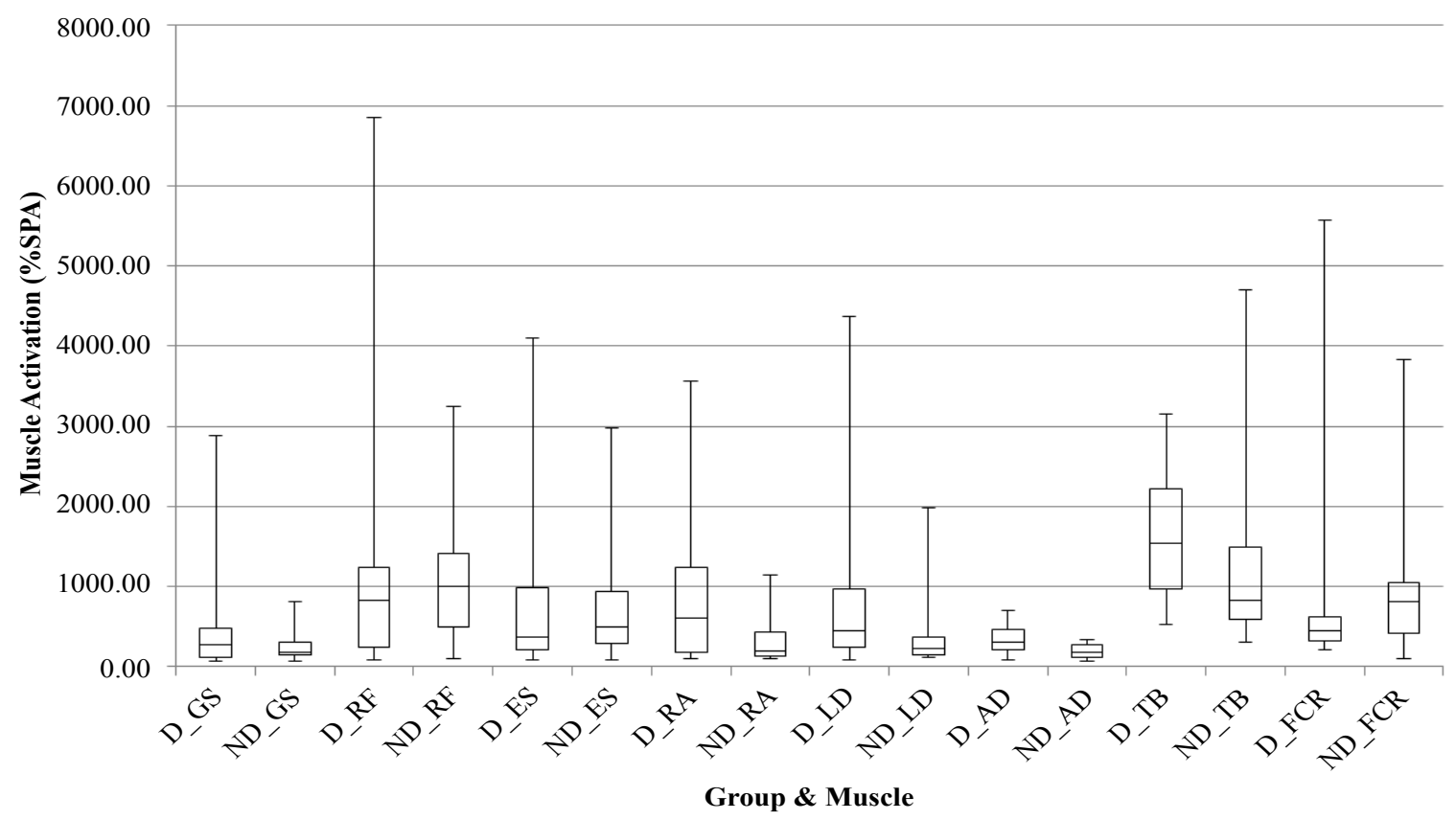

Figure 1a: Boxplots illustrating non-normality of raw EMG data for each muscle within both the Disability and No Disability groups.

D = Disability; ND = No Disability; GS = Gastrocnemius; RF = Rectus Femoris; ES = Erector Spinae; LD = Latissimus Dorsi; $\mathrm{AD}=$ Anterior Deltoid; $\mathrm{MT}=$ Triceps Brachii; FCR = Flexor Carpi Radialis. 


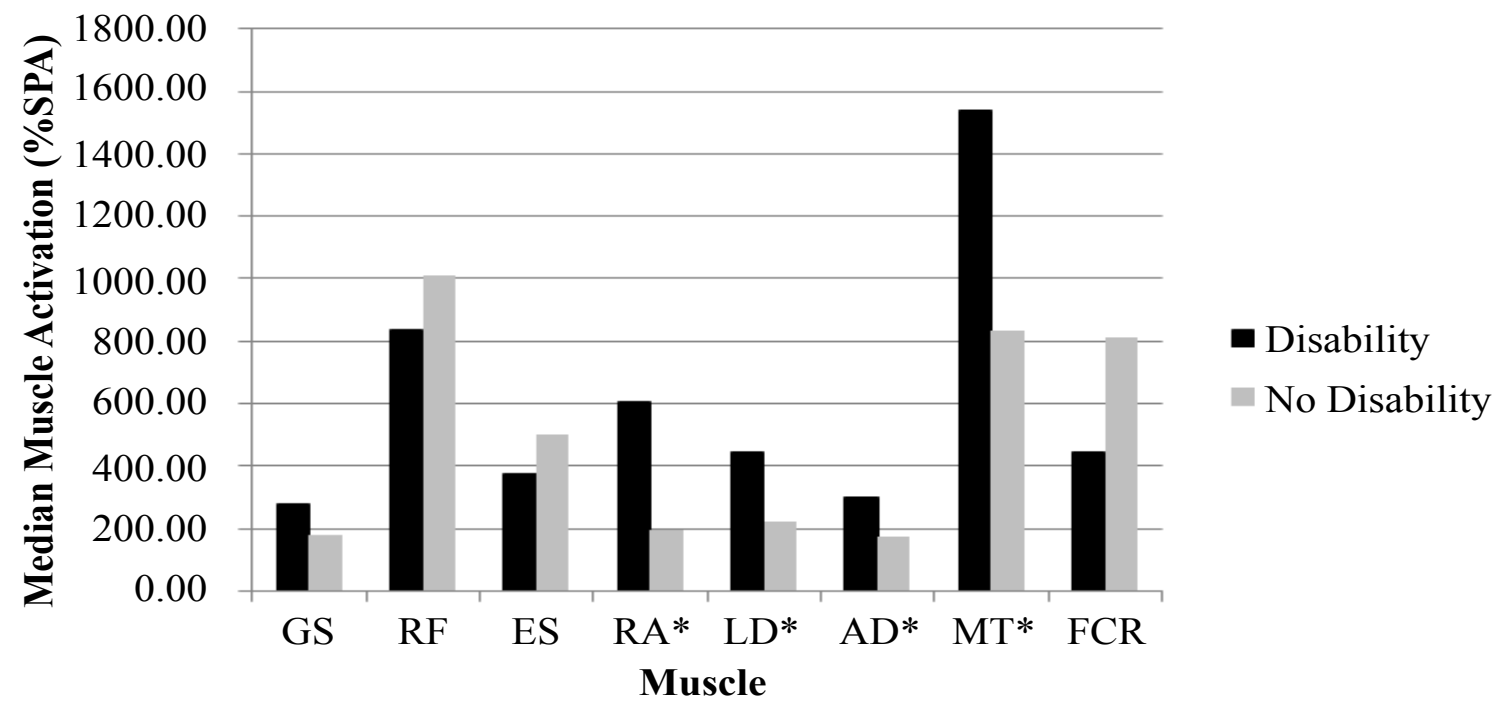

Figure 1b: Raw EMG data for each muscle expressed as \%SPA for each group.

GS = Gastrocnemius; RF = Rectus Femoris; $E S=$ Erector Spinae; LD = Latissimus Dorsi; AD = Anterior Deltoid; MT = Triceps Brachii; FCR = Flexor Carpi Radialis; ${ }^{*}$ - indicates statistical significance $(p<0.05)$.

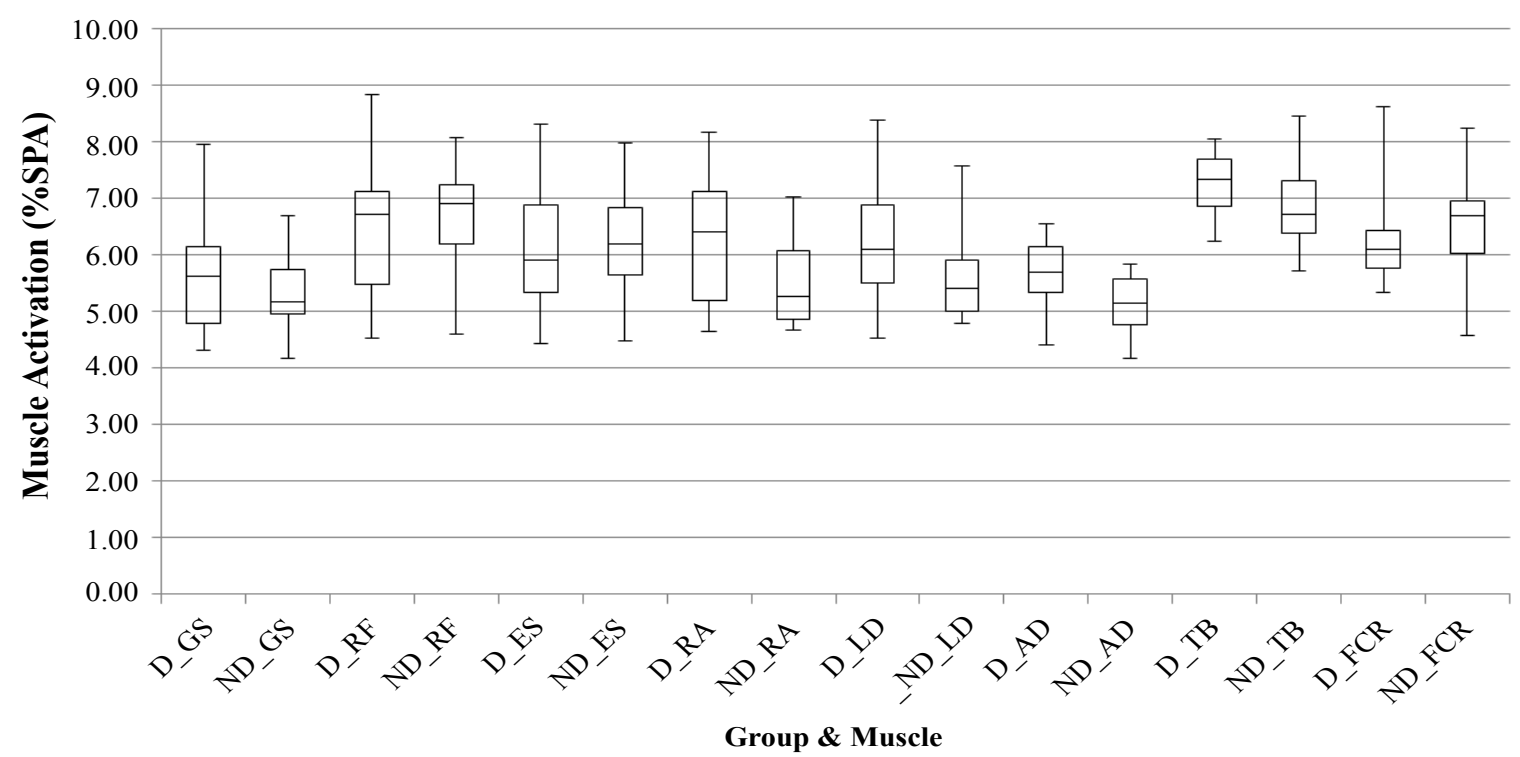

Figure 1c: Boxplots illustrating normality of natural log transformed EMG data for each muscle within both the Disability and No Disability groups.

D = Disability; ND = No Disability; GS = Gastrocnemius; RF = Rectus Femoris; ES = Erector Spinae; LD = Latissimus Dorsi; $A D=$ Anterior Deltoid; MT = Triceps Brachii; FCR = Flexor Carpi Radialis.

were found for the rectus abdominis (608.0 vs. 195.0 $\% S P A, p=0.025)$, latissimus dorsi (446.0 vs. $224.3 \%$ SPA, $p=0.022$ ), anterior deltoid (300.3 vs. $173.7 \%$ SPA, $p=$ 0.003 ), and triceps brachii (1537.7 vs. $832.7 \%$ SPA, $p=$ 0.031 ), with participants with disabilities yielding greater muscle activation in the four aforementioned muscles compared to the participants without disabilities. Similarly, independent samples t-test using natural log transformed normal data found significant mean differences in muscle activation between participants with and without disabilities were found for the rectus abdominis (6.21 vs. $5.52 \% \mathrm{SPA}, \mathrm{p}=0.019$ ), latissimus dorsi (6.12 vs. $5.58 \%$ SPA, $p=0.031$ ), anterior deltoid (5.59 vs. $5.12 \% \mathrm{SPA}, p=0.003$ ), and triceps brachii (7.26 vs. $6.87 \%$ SPA, $p=0.046)$, with participants with disabilities yielding greater muscle activation in the four aforementioned muscles compared to the participants without disabilities. All remaining muscle activation EMG data were not significantly different between participants with and without disabilities.

\section{Discussion}

The purpose of this study was to identify muscle activations of commonly used lumbopelvic-hip complex (erector spinae, rectus abdominis, and latissimus dorsi), lower extremity (gastrocnemius and rectus femoris), and upper extremity (anterior deltoid, triceps brachii, and flexor carpi radialis) musculature in wheelchair basketball free throw shooting to better understand energy transfer in wheelchair basketball players with disabili- 


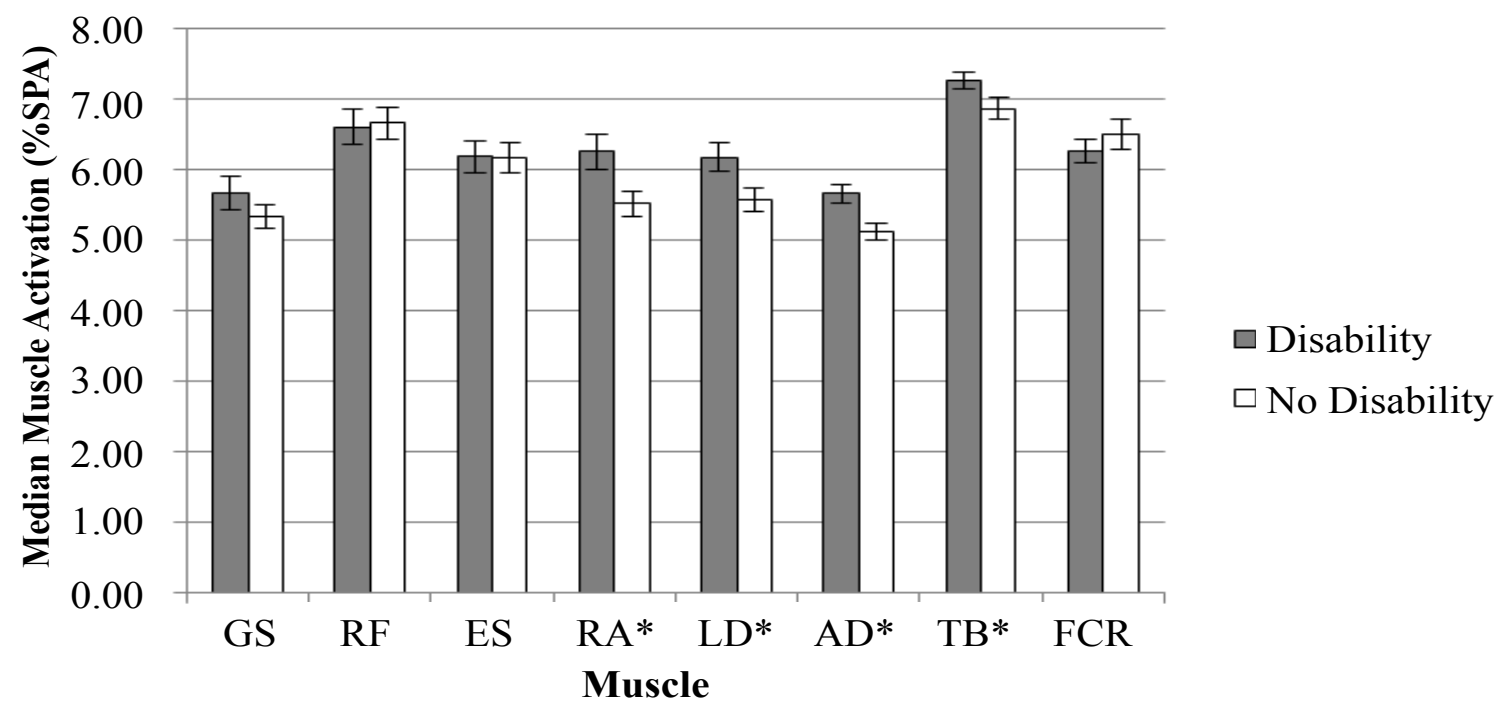

Figure 1d: Natural log transformed EMG data for each muscle expressed at \%SPA for each group.

GS = Gastrocnemius; RF = Rectus Femoris; $E S=$ Erector Spinae; LD = Latissimus Dorsi; $A D=$ Anterior Deltoid; $M T=$ Triceps Brachii; FCR = Flexor Carpi Radialis; * ${ }^{*}$ indicates statistical significance $(p<0.05)$.

ties compared to basketball players without disabilities shooting from a wheelchair. Understanding muscle activations in commonly used musculature in the free throw is important for the development of strength and functional training to increase shot performance. Findings from this research study revealed that wheelchair basketball athletes with a disability have significantly greater activation of the rectus abdominis, latissimus dorsi, anterior deltoid, and triceps brachii muscles compared to wheelchair basketball athletes without a disability when shooting a free throw (Figure $1 b$ and Figure $1 c)$. With significant differences of muscle activation in these muscles, these findings suggest that wheelchair basketball athletes with disabilities require greater muscle activation in select muscles to perform an accurate free throw shooting task.

As the results of the study indicate, the rectus abdominis, latissimus dorsi, anterior deltoid, and medial triceps are greatly involved in the free throw shooting motion in wheelchair basketball athletes with disabilities. Depending on the extent of the disability, the importance of the rectus abdominis is multifaceted. For those who have affected lower extremities but have retained core control, such as amputees, the rectus abdominis may be important for trunk flexion to initiate the shot in the frontal plane. For individuals who do not have typical core control, the rectus abdominis is important for maintaining a proper center of mass to avoid losing balance while shooting. The latissimus dorsi may also be important in the shooting motion. Wheelchair basketball athletes with a disability may utilize this muscle when bracing themselves against the backrest of the wheelchair while moving their shooting shoulder downward into an extended and adducted position during the countermovement before the shot. Similarly, the anterior deltoid is likely relied upon for shoulder flexion in the frontal plane during shooting. This is possibly the most commonly used muscle when shooting, as the anterior deltoid places the upper arm and shoulder into an elevated position to apply force to the underside of the basketball and allow for optimal trajectory required to execute an accurate shot. As previously investigated, athletes with disabilities, specifically those with less function, perform a free throw from a lower seated position requiring a steeper shot trajectory, theoretically requiring greater activation and force production from the shoulder flexors, such as the anterior deltoid. Lastly, the medial triceps brachii are vital during elbow extension associated with the follow-through of shooting a basketball [20]. Similarly, because wheelchair basketball athletes with disabilities tend to use greater upper extremity musculature to generate a forceful shot, it is likely that greater triceps brachii activation is necessary to perform an accurate shot.

A limitation of the current study was the utilization of experienced basketball players without a disability having inexperience shooting free throws from a competition wheelchair. Due to the decreased level of experience in performing a free throw without the use of their lower extremities, as well as significant differences in shot trajectory compared to standing basketball, these individuals likely used inconsistent shot mechanics, thereby affecting muscle activation. Lastly, a final limitation of the current study was the analysis of wheelchair basketball players with a disability as a collective group. Future research should analyze differences in muscle activation patterns based on physical disability or by IWBF classification (iwbf.org) to further explain differences in muscle activation across multiple levels of wheelchair basketball players with varying disabilities.

\section{Conclusion}

The results of the current study showed significant 
differences in muscle activation intensities between wheelchair basketball players with and without disabilities. Experienced wheelchair basketball players with disabilities showed greater activation of the shooting-side rectus abdominis, latissimus dorsi, anterior deltoid and medial triceps brachii muscles compared to experienced basketball players without a disability. These findings suggest that wheelchair basketball players with disabilities maintain summation of proximal to distal sequencing using the lumbopelvic-hip complex as the origin of movement, rather than the lower extremities, which are rendered motionless due to strapping and bracing components of the wheelchair. From a performance standpoint, these findings may provide insight into the discrepancy between free throw shooting percentages between able-bodied basketball players and wheelchair basketball players. Future research should investigate this by identifying alterations in muscle activations between these two populations.

These results also suggest that the trunk and upper extremity muscles are primarily responsible for movements and sport-specific tasks in wheelchair basketball players with disabilities. This is self-explanatory given the nature of the sport; requiring muscle activation from the trunk and upper extremities to perform all aspects of the sport (wheelchair propulsion, dribbling, passing, and shooting). This could suggest why previous research has found upper extremity pain to be one of the most common injuries in wheelchair users, in both athletic and non-athletic populations, accounting for $33-85 \%$ of all reported pain [21-26].

These findings may assist sports medicine professionals in understanding the cumulative stresses of competitive wheelchair usage and participating in an overhead sport, such as wheelchair basketball. By identifying muscles of the trunk and upper extremities that are largely responsible for movement and task performance, strength coaches, physical therapists, and athletic trainers may assist them to modify exercises and interventions to strengthen these muscles to increase shooting performance, as well as target the functional synergists and antagonists to restore muscle balance and prevent chronic upper extremity injuries.

Auburn University Institutional Review Board approved the study protocol. The authors certify that they have no affiliations with or financial involvement in any organization or entity with a direct financial interest in the subject matter or materials discussed in the manuscript.

\section{References}

1. Nunome H, Doyo W, Sakurai S, Ikegmai Y, Yabe K (2002) A kinematic study of the upper-limb motion of wheelchair basketball shooting in tetraplegic adults. J Rehabil Res Dev 39: 63-71.

2. Malone LA, Gervais PL, Steadward RD (2002) Shooting mechanics related to player classification and free throw success in wheelchair basektball. J Rehabil Res Dev 39: 701-709.
3. Ingram B, Snowden S (1989) Face up to good shooting technique. Scholastic Coach 59: 58-59.

4. Ogden L (2015) Why Are Able-Bodied People Playing Wheelchair Basketball?

5. Malone LA, Nielsen AB, Steadward RD (2000) Expanding the dichotomous outcome in wheelchair basketball shooting of elite male players. Adapted Physical Activity Quarterly 17: 437-449.

6. NBA (2017) NBA Advanced Stats.

7. IWBF (2017) Statistics.

8. Schwark BN, Mackenzie SJ, Sprigings EJ (2004) Optimizing the release conditions for a free throw in wheelchair basketball. Journal of Applied Biomechanics 20: 153-166.

9. IWBF (2014) Official Player Classification Manual.

10. Oliver GD, Plummer HA, Keeley DW (2011) Muscle activation patterns of the upper and lower extremity during the windmill softball pitch. J Strength Cond Res 25: 1653-1658.

11. Plummer HA, Oliver GD (2014) The relationship between gluteal muscle activation and throwing kinematics in baseball and softball catchers. J Strength Cond Res 28: 87-96.

12. Putnam CA (1991) A segment interaction analysis of proximal-to-distal sequential segment motion patterns. Med Sci Sports Exerc 23: 130-144.

13. Putnam CA (1993) Sequential motions of body segments in striking and throwing skills: Descriptions and explanations. J Biomech 26: 125-135.

14. Qi L, Wakeling J, Grange S, Ferguson Pell M (2013) Coordination patterns of shoulder muscles during level-ground and incline wheelchair propulsion. J Rehabil Res Dev 50: 651-662.

15. Uzun S, Pourmoghaddam A, Hieronymus $M$, Thrasher $T$ (2012) Evaluation of muscle fatigue of wheelchair basketball players with spinal cord injury using recurrence quantification analysis of surface EMG. Eur J Appl Physiol 112: 3847-3857.

16. Yang YS, Koontz AM, Triolo RJ, Mercer JL, Boninger ML (2006) Surface electromyography activity of trunk muscles during wheelchair propulsion. Clin Biomech 21: 1032-1041.

17. Oliver GD, Stone AJ, Plummer H (2010) Electromyographic examination of selected muscle activation during isometric core exercises. Clin J Sport Med 20: 452-457.

18. Halaki M, Ginn K (2012) Normalization of EMG signals: To normalize or not to normalize and what to normalize to? In: Computational intelligence in electromyography analysis- $A$ perspective on current applications and future challenges. Intech.

19. Cram JR (1998) Introduction to surface electromyography. Aspen Publishers.

20. Pakosz P (2011) EMG signal analysis of selected muscles during shots and passes in basketball. $\mathrm{J}$ Health Promotion Recreation 1: 9-14.

21. Ambrosio F, Boninger ML, Souza AL, Fitzgerald SG, Koontz A, et al. (2005) Biomechanics and strength of manual wheelchair users. J Spinal Cord Med 28: 407-414.

22. Ballinger DA, Rintala DH, Hart KA (2000) The relation of shoulder pain and range-of-motion problems to functional limitations, disability and perceived health of men with spinal cord injury: A multifaceted longitudinal study. Arch Phys Med Rehabil 81: 1575-1581. 
23. Curtis K, Roach K, Applegate EB, Amar T, Benbow CS, et al. (1995) Development of the wheelchair user's shoulder pain index (WUSPI). Spinal Cord 33: 290-293.

24. Curtis KA, Drysdale GA, Lanza RD, Kolber M, Vitolo RS, et al. (1999) Shoulder pain in wheelchair users with tetraplegia and paraplegia. Arch Phys Med Rehabil 80: 453-457.
25. Roach KE, Budiman Mak E, Songsiridej N, Lertratanakul Y (1991) Development of a shoulder pain and disability index. Arthritis \& Rheumatology 4: 143-149.

26. Salisbury SK, Choy NL, Nitz J (2003) Shoulder pain, range of motion and functional motor skills after acute tetraplegia. Arch Phys Med Rehabil 84: 1480-1485. 